\title{
Contaminación biológica con heces caninas y parásitos intestinales en espacios públicos urbanos en dos ciudades de la Provincia del Chubut. Patagonia Argentina
}

\author{
PAULA SÁNCHEZ*, SILVINA RASO*, CLAUDIA TORRECILLAS*, IVANA MELLADO*, \\ ADRIÁN ÑANCUFIL**, CINTIA M. OYARZO**, MARÍA E. FLORES*, MIRTA CÓRDOBA*, \\ MARTA C. MINVIELLE*** y JUAN A. BASUALDO***
}

\section{BIOLOGICAL CONTAMINATION WITH CANINE FAECES AND INTESTINAL PARASITES IN PUBLIC PARKS IN TWO CITIES OF CHUBUT PROVINCE, PATAGONIA ARGENTINA}

A longitudinal study to determine the presence of intestinal parasites in canine faeces and their relationship with seasonal weather conditions during a year was carried out. 481 fecal canine deposits were collected from 13 public parks in two cities of Chubut Province, Patagonia Argentina. Stools were analyzed by means of Telemann and Willis methods. Weather conditions were daily registered from local stations. The frequency of positive canine faeces to intestinal parasites was found between $34,9 \%$ and 51,2\% over the studied period and was season independent $(p>0.05)$. A predominance of helminths over protozoa was detected in all seasons. Frequency of Entamoeba spp. and Nematoda was seasonal dependent $(p<0.05)$. The finding of Toxocara canis eggs varied from 12,7\% in winter to 20,9\% in spring. Pathogens to humans such as T. canis, Taenia spp., Uncinaria spp. and Entamoeba spp. were found. Spirocerca spp. and Capillaria spp. have been reported for the first time for this region.

Key words: Contamination, Canine faeces, Intestinal parasites, Argentine.

\section{INTRODUCCIÓN}

Las parasitosis podrían considerarse como el resultado o expresión de un tipo de articulación en espacio y tiempo, entre el hombre y su ambiente ${ }^{1}$.

El primer eslabón en la secuencia de transmisión de algunas zoonosis parasitarias es la exposición del hombre a huevos, quistes y ooquistes infectivos de helmintos y protozoos ${ }^{2-}$ ${ }^{7}$. Dicha exposición está influenciada por factores culturales, de comportamiento (hábitos de defecación de mascotas) y climáticos que condicionan la dispersión y persistencia de parásitos en el ambiente ${ }^{8,9}$.

Los espacios públicos urbanos, constituyen

* Dept. de Bioquímica. Facultad de Ciencias Naturales. Universidad Nacional de la Patagonia San Juan Bosco. Ciudad Universitaria. Km. 4. Comodoro Rivadavia. C.P. 9000. Chubut. Argentina.

** Dept.de Geografía. Fac. de Humanidades. Universidad Nacional de la Patagonia San Juan Bosco.

***Cátedra de Microbiología y Parasitología. Facultad de Ciencias Médicas. Universidad Nacional de La Plata. 60 y 120. La Plata. C.P. 1900. Buenos Aires. Argentina.

e-mail: psanchez@unpata.edu.ar 
un lugar de recreación para los habitantes de la ciudad $^{9}$. La contaminación biológica de éstos espacios con materia fecal canina conteniendo formas parasitarias infectantes, es un factor de riesgo para niños y adultos ${ }^{10}$.

La influencia de factores físicos sobre la viabilidad y desarrollo de los parásitos en el ambiente se ha demostrado a través de diversos estudios experimentales. Se ha reportado una supervivencia de huevos de Taenia spp. por períodos de 300 días a temperaturas entre $0^{\circ} \mathrm{C}$ $10^{\circ} \mathrm{C}$ con $85 \%$ de humedad relativa ${ }^{11,12}$. Temperaturas inferiores a $20^{\circ} \mathrm{C}$ o superiores a $40^{\circ} \mathrm{C}$ inhiben el proceso de esporulación de ooquistes de Isospora spp. obtenidos de perros infectados, siendo las condiciones óptimas que permiten este proceso las temperaturas comprendidas entre $30^{\circ} \mathrm{C}$ y $37^{\circ} \mathrm{C}^{5}$. Los quistes de Giardia spp. pueden sobrevivir a bajas temperaturas y humedad.

Los objetivos de éste trabajo fueron determinar la presencia de parásitos intestinales en muestras de heces caninas recolectadas en las plazas de las ciudades de Comodoro Rivadavia y Rada Tilly (Provincia del Chubut - Patagonia Argentina) y su relación con las condiciones climáticas estacionales durante un año.

\section{MATERIAL Y MÉTODO}

Las ciudades de Comodoro Rivadavia y Rada Tilly están ubicadas sobre la costa patagónica, en el extremo sur-este de la Provincia del Chubut
- Patagonia Argentina $\left(45^{\circ} \mathrm{S} 68^{\circ} \mathrm{O}\right)$, distantes $20 \mathrm{~km}$ una de la otra.

Para el presente estudio se seleccionaron 3 de las 5 plazas de la ciudad de Rada Tilly y 10 de las 55 plazas de la ciudad de Comodoro Rivadavia. Las plazas fueron estudiadas longitudinalmente durante las distintas estaciones del año, entre julio 1999 y junio 2000. En cada plaza se censó el número total de heces halladas y se recolectaron al azar, en un muestreo por conglomerados, 10 muestras de materia fecal canina.

Las heces fueron examinadas macroscopicamente y fijadas con formaldehído $5 \%$. Fueron concentradas por medio de la técnica de flotación de Willis y de sedimentación de Telemann y observadas por duplicado mediante microscopía óptica con y sin lugol.

Los datos meteorológicos de temperatura, lluvia y vientos se recopilaron a partir de los registros diarios de la Estación Meteorológica Davies, la Estación portuaria de la Base de Información Georeferenciada de la Universidad Nacional de la Patagonia San Juan Bosco ${ }^{13,14}$.

El análisis estadístico de los datos obtenidos se efectuó aplicando la prueba de independencia de Chi-cuadrado y la prueba de Homogeneidad para análisis de proporciones. Se tomó como valor de aceptación de hipótesis nula un valor de $\mathrm{p}>0,05$.

\section{RESULTADOS}

Durante el período estudiado (julio1999 / junio 2000) se censó un total de 1.855 heces caninas

Tabla 1. Distribución estacional de la contaminación con heces caninas en plazas de Comodoro Rivadavia y Rada Tilly, Chubut, julio 1999/junio 2000

\begin{tabular}{lrrrr}
\hline & Invierno & Primavera & Verano & \multicolumn{1}{c}{ Otoño } \\
\hline $\mathrm{N}^{\circ}$ de heces halladas/ $\mathrm{N}^{\mathbf{o}}$ plazas/estudiadas & $728 / 13$ & $204 / 13$ & $254 / 12$ & $669 / 13$ \\
$\begin{array}{l}\text { Promedio de } \mathrm{N}^{\circ} \text { de heces halladas/plaza } \\
\text { (mínimo - máximo) }\end{array}$ & 56 & 16 & 21 & 51 \\
\hline
\end{tabular}

Tabla 2. Frecuencia de heces positivas para parásitos en plazas de Comodoro Rivadavia y Rada Tilly, Chubut, julio 1999/junio 2000

\begin{tabular}{lrrrr}
\hline & Invierno & Primavera & Verano & Otoño \\
\hline $\mathrm{N}^{\circ}$ de heces estudiadas (481) & 110 & 129 & 116 & 126 \\
$\mathrm{~N}^{\circ}$ y \% de muestras de heces positivas & 42 & 66 & 49 & 44 \\
para parásito & $38,2 \%$ & $51,2 \%$ & $42,2 \%$ & $34,9 \%$ \\
$\mathrm{~N}^{\circ}$ y \% de muestras de heces & 10 & 21 & 19 & 15 \\
poliparasitadas & $9,1 \%$ & $16,3 \%$ & $16,4 \%$ & $11,9 \%$ \\
\hline
\end{tabular}


en las 13 plazas muestreadas; con un mínimo de 4 y un máximo de 229 heces caninas/plaza. En la Tabla 1 se muestra la distribución por estación de la contaminación por heces caninas de los espacios públicos estudiados.

De las heces halladas, se analizaron 481 $(25,9 \%)$, resultando positivas para parásitos intestinales 201 heces $(41,8 \%)$, presentando 65 heces $(13,5 \%)$ más de un género de parásitos (Tabla 2). El hallazgo de heces caninas positivas para parásitos intestinales resultó independiente de la estación $(\mathrm{p}>0,05)$.

En la Tabla 3 se presenta el género y frecuencia de parásitos intestinales encontrados, y su distribución por estación. Del análisis estadístico de las proporciones individuales de cada género detectado, comparadas por estación, surge que el hallazgo de Entamoeba spp. y larvas de Nematoda es dependiente de la estación del año $(\mathrm{p}<0,05)$.
Durante las cuatro estaciones estudiadas la frecuencia de detección de helmintos fue superior a protozoos, resultando significativa la diferencia $(\mathrm{p}<0,05)$.

Taenia spp., Toxocara canis y larvas de Nematoda se recuperaron durante las cuatro estaciones estudiadas; siendo éstos dos últimos los parásitos que presentan mayor frecuencia de aparición. Spirocerca spp. y Uncinaria spp. no se detectaron en invierno; y Diphylidium caninun y Capillaria spp. sólo se hallaron en una estación (invierno y primavera respectivamente), en baja frecuencia.

Del total de huevos de T. canis hallados el $14,28 \%, 40,74 \%, 20,09 \%$ y $39,13 \%$ se presentaron larvados (larvas en fase II), para invierno; primavera; verano y otoño respectivamente.

Entre los protozoos, Isospora spp. se detectó en las cuatro estaciones. Entamoeba spp. no se

Tabla 3. Frecuencia de parásitos intestinales hallados en muestras de materia fecal canina recolectadas en plazas de Comodoro Rivadavia y Rada Tilly, Chubut, (julio 1999/junio 2000). N = 481

\begin{tabular}{|c|c|c|c|c|c|c|c|c|c|}
\hline & \multicolumn{2}{|c|}{ Invierno } & \multicolumn{2}{|c|}{ Primavera } & \multicolumn{2}{|c|}{ Verano } & \multicolumn{2}{|c|}{ Otoño } & \multirow[t]{2}{*}{$\mathbf{P}$} \\
\hline & $\mathbf{N}$ & $\%$ & $\mathbf{N}$ & $\%$ & $\mathbf{N}$ & $\%$ & $\mathbf{N}$ & $\%$ & \\
\hline Muestra analizadas & 110 & 100 & 100 & 129 & 116 & 100 & 126 & 100 & \\
\hline Protozoos & 6 & 5,5 & 11 & 8,5 & 27 & 23,3 & 14 & 11,1 & \\
\hline Isospora $\mathrm{sp}$ & 4 & 3,6 & 4 & 3,1 & 3 & 2,6 & 1 & 0,8 & 0,5150 \\
\hline Sarcocystis sp & - & - & - & - & 2 & 1,7 & - & - & 0,09 \\
\hline Entamoeba sp & - & - & 7 & 5,4 & 21 & 18,1 & 11 & 8,73 & 0,00007 \\
\hline Endolimax sp & 1 & 0,9 & - & - & - & - & - & - & 0,3367 \\
\hline Giardia sp & 1 & 0,9 & - & - & 1 & 0,9 & 2 & 1,59 & 0,5799 \\
\hline Helmintos & 40 & 36,4 & 80 & 62 & 51 & 44 & 46 & 36,5 & \\
\hline Toxocara canis & 14 & 12,7 & 27 & 20,9 & 23 & 19,8 & 23 & 18,3 & 0,38 \\
\hline Nematoda sp & 22 & 20 & 39 & 30,2 & 15 & 12,9 & 18 & 14,3 & 0,00193 \\
\hline Uncinarias sp & - & - & 2 & 1,6 & 2 & 1,7 & 1 & 0,8 & 0,5549 \\
\hline Spirocerca sp & - & - & 5 & 3,9 & 5 & 4,3 & 3 & 2,4 & 0,1777 \\
\hline Capillara sp & - & - & 1 & 0,8 & - & - & - & - & 0,4344 \\
\hline Taenia sp & 4 & 3,6 & 6 & 4,7 & 6 & 5,2 & 1 & 0,8 & 0,2442 \\
\hline D. caninum & 1 & 0,9 & - & - & - & - & - & - & 0,5511 \\
\hline
\end{tabular}

Tabla 4. Datos del clima en Comodoro Rivadavia (julio 1999/junio 2000). Valores medios

\begin{tabular}{|c|c|c|c|c|}
\hline & Invierno & Primavera & Verano & Otoño \\
\hline Temperatura media & 8,0 & 15,5 & 20,4 & 14,7 \\
\hline Precipitaciones (mm) & 13,4 & 22,2 & 2,3 & 25,7 \\
\hline Temperatura máxima absoluta $\left({ }^{\circ} \mathrm{C}\right)$ & 19,0 & 28,3 & 33,2 & 24,9 \\
\hline Temperatura mínima absoluta $\left({ }^{\circ} \mathrm{C}\right)$ & $-2,7$ & 5,2 & 10,6 & 7,0 \\
\hline Velocidad del viento $(\mathrm{kph})$ & 20,6 & 16,0 & 14,1 & 7,4 \\
\hline Dirección del viento predominante & oeste & oeste & oeste & oeste \\
\hline
\end{tabular}


encontró en invierno y presentó un máximo de incidencia en verano $(18,10 \%)$. El resto de los protozoos hallados (Sarcocystis spp., Giardia spp., Endolimax spp.), se presentaron en forma inconstante y en baja frecuencia durante las distintas estaciones.

Las formas parasitarias recuperadas fueron: quistes (Entamoeba spp., Giardia spp., Endolimax spp.), ooquistes (Isospora spp.), esporoquistes (Sarcocystis spp.), huevos larvados o embrionados (Toxocara spp., Capillaria spp., Spirocerca spp., Uncinaria spp., Taenia spp., D. caninun) y larvas (Nematoda).

En Tabla 4 se resumen los datos de las variables climáticas para cada estación. Los períodos de registro de mayor temperatura coincidieron con los períodos de registro mínimo de precipitaciones (noviembre-abril). La mínima temperatura registrada durante el período estudiado fue de $-3^{\circ} \mathrm{C}$ en invierno y la máxima de $35,3^{\circ} \mathrm{C}$ en verano. El viento se presentó en forma constante durante todo el año, con períodos de mayor intensidad durante invierno-primavera e inicio del verano, siendo la dirección predominante: oeste.

\section{DISCUSIÓN}

Los resultados obtenidos demuestran elevada contaminación de las plazas estudiadas con materia fecal canina. De los distintos géneros detectados, $9(90 \%)$ tienen la capacidad potencial de infectar al hombre (Isospora, Sarcocystis, Entamoeba, Giardia, Endolimax, Toxocara, Capillaria, Taenia y Uncinaria).

Las características variables de las condiciones meteorológicas registradas en las estaciones estudiadas condicionarían la presencia de algunos de los parásitos intestinales hallados, tales como Entamoeba spp. y Nematoda. Estas características, actuarían como factores extrínsecos que regulan la estabilidad del parásito en el ambiente ${ }^{4,15}$. La condición de desecación originada por diferentes causas: altas temperaturas y bajo régimen de precipitaciones en verano; aumento en la frecuencia e intensidad de los vientos en invierno y primavera, constituye el factor físico predominante a lo largo del año que puede ejercer un efecto deletéreo sobre algunos géneros de parásitos $^{5,7,9,10,12,15}$. Estudios experimentales han demostrado la resistencia de Nematoda a la desecación, los cuales tienen la capacidad de entrar en un estado de dormancia o anhidrobiosis como mecanismo adaptativo ante condiciones extremas de deshidratación ${ }^{16,17}$. Esto podría explicar la presencia de larvas de Nematoda durante las distintas estaciones del año.

Las bajas temperaturas prevalentes en invierno, inferiores a $0^{\circ} \mathrm{C}$, determinarían la ausencia de parásitos propios de climas cálidos tales como Spirocerca spp.; Capillaria spp y Uncinaria spp. La aparición de éstos géneros en las estaciones de mayor registro térmico apoyan ésta hipótesis.

La presencia de T. canis e Isospora spp. en las heces caninas sumado a la ausencia de campañas de esterilización desde el año 1998, podría indicar el estado y tipo de población canina que visita las plazas estudiadas. Se ha reportado una mayor prevalencia de infección en cachorros y hembras preñadas con $T$. canis y una mayor susceptibilidad a la infección con Isospora spp. de los cachorros y perros vagabundos ${ }^{3,5,18}$. Con relación a Isospora spp., el estrés del destete y una mayor exposición a hospederos paraténicos infectados, debido a la necesidad de cazar para alimentarse de los perros sin dueño, origina la mayor susceptibilidad de éste grupo ${ }^{5}$. El desarrollo de inmunidad posterior a una primoinfección, sin descarga subsecuente de ooquistes ante nuevos desafíos, explicaría los valores relativamente bajos de incidencia de Isospora spp. a través del tiempo.

Se destaca el hallazgo de huevos del género de Taenia spp. en la materia fecal de las plazas estudiadas, considerando que nuestra provincia es una zona endémica en áreas rurales para hidatidosis. La detección en el ámbito urbano de éstos elementos infectantes, independientemente de la taxonomía de dichos huevos, podría representar la instalación del ciclo y conductas de riesgo en las ciudades estudiadas; probablemente debido al fenómeno social de ruralización de las mismas y/o influencia del ciclo silvestre de la enfermedad ${ }^{19}$.

Los resultados de éste estudio demuestran elevada contaminación con heces caninas de los espacios públicos de uso recreacional en las ciudades estudiadas. Esta contaminación está influenciada por factores climáticos.

Los porcentajes de muestras positivas para parásitos intestinales de materia fecal canina recolectadas en las plazas estudiadas resultaron elevados e independientes de las estaciones ( $\mathrm{p}>$ $0,05)$. Se registró predominio de helmintos en 
todas las estaciones estudiadas.

El hallazgo de Entamoeba spp. y Nematoda resultó dependiente de la estación del año $(\mathrm{p}<0,05)$.

Se reportan por primera vez los géneros Capillaria y Spirocerca para la zona. Se encontraron parásitos patógenos para el humano tales como Toxocara spp., Taenia spp., Uncinaria spp. y Entamoeba spp.

El hallazgo de huevos de Taenia spp. en los espacios públicos recreacionales; justifica la implementacion de medidas de control adecuadas a zonas urbanas, en zonas endémicas para hidatidosis, como la Provincia del Chubut.

\section{RESUMEN}

Se realizó un estudio longitudinal durante un año, para determinar la presencia de parásitos intestinales en heces caninas y su relación con las condiciones climáticas. 481 muestras de heces fueron recolectadas en 13 plazas de dos ciudades de la Provincia del Chubut, Patagonia Argentina. Las muestras fueron analizadas por medio de los métodos de Telemann y Willis. Los datos meteorológicos se registraron diariamente. La frecuencia de aparición de muestras positivas para parásitos intestinales estuvo comprendida entre el $34,9 \%$ y $51,2 \%$ durante el periodo estudiado, resultando la misma independiente de la estación climática $(p>0,05)$. Se registró un predominio de helmintos sobre protozoos durante las cuatro estaciones. La frecuencia de aparición de Entamoeba spp. y Nematoda resultó dependiente de la estación estudiada $(\mathrm{p}<0,05)$. El hallazgo de huevos de $T$. canis varió de un $12,7 \%$ en invierno a un $20,9 \%$ en primavera. Se detectaron patógenos para el humanos como T. canis, Taenia spp., Uncinaria spp. y Entamoeba spp. Se reportan por primera vez para ésta región Spirocerca spp. y Capillaria spp.

\section{REFERENCIAS}

1.- LAHITTE H B, HURREL J A, MALPARTIDA M A. Relaciones. Crítica y expansión de la ecología de las ideas. Edit. Nuevo Siglo. 1989. pp: 231.

2.- WOLFE M S. Giardiasis. Clin. Microbiol. Rev 1992; Jan 93-100.

3.- ATIAS A. Parasitología Clínica. Ed. Mediterráneo. 1994; pp: 547-57.

4.- THOMPSON R C A, LYMBERY A J. Echinococcus and hydatid disease. CAB International Ed. 1995; pp: 1-37.

5.- LINDSAY D S, DUBEY J P, BLAGBURN B L. Biology of Isospora spp. from humans, nonhuman primates and domestic animals. Clin Microbiol Rev 1997; Jan: 19-34.

6.- CLARK D P. New insights into human Cryptosporidiosis. Clin Microbiol Rev 1999; Oct: 554-63.

7.- EL-SHEHABI F S, ABDEL-HAFEZ S K, KAMHAWI S A. Prevalence of intestinal helminths of dogs and foxes from Jordan. Parasitol Res 1999; 85: 928-34.

8.- UGA S, MINAMI T, NAGATA K. Defecation habits of cats and dogs and contamination by Toxocara eggs in public park sandpits. Am J Trop Med Hyg 1996; 54 : 122-6.

9.- KITHTLINGER L K, SEED J R, KITHTLINGER M B. Ascaris lumbricoides intensity in relation to environmental, socioeconomic, and behavioral determinants of exposure to infection in children from southeast Madagascar. J Parasitol 1998; 84: 480-4.

10.- MINVIELLE M C, PEZZANI B C, BASUALDO J A. Frecuencia de hallazgo de huevos de helmintos en materia fecal canina recolectada en lugares públicos de la ciudad de La Plata, Argentina. Bol Chil Parasitol. 1993; 48: 63-5.

11.- ILSOE B, KYVSGAARD N CH, NANSEN P, HENRIKSEN S A. A study on the survival of Taenia saginata eggs on soil in Denmark. Acta Vet Scand 1990; 31: 153-8.

12.- WACHIRA T M, MACPHERSON C N L, GATHUMA J M. Release and survival of Echinococcus eggs in different environments in Turkana, and their possible impact on the incidence of hydatidosis in man and livestock. J Helminthol 1991; 65: 55-61.

13.- BURCHERI A M. Datos climatológicos Estación Meteorológica Portuaria. Base de Información Georeferenciada. Universidad Nacional de la Patagonia San Juan Bosco. Datos no publicados. 1999. Comunicación personal.

14.- RAIMONDI A. Datos climatológicos Estación Meteorológica Davies. Liceo Militar General Roca. Comodoro Rivadavia.Chubut. Datos no publicados. 2000. Comunicación personal.

15.- CABRERA P A, HARAN G, BENAVIDEZ U et al. Transmission dynamics of Echinococcus granulosus, Taenia hydatígena and Taenia ovis in sheep in Uruguay. Int J Parasitol 1995; 25: 807-13.

16.- CROWE J H, MADIN K A C. Anhydrobiosis in Nematodes: evaporative water loss and survival. J Exp Zool 1976; 193: 323-34.

17.- WHARTON D A, LEMMON J. Ultraestructural changes during desiccation of the anhydrobiotic nematode Ditylenchus dipsaci. Tissue \& Cell 1998; 30: 312-23.

18.- PEREIRA D L, BASUALDO J A, MINVIELLE M C et al. Catastro parasitológico. Helmintiasis en canes. Área: Gran La Plata. Sobre 1.000 casos. Vet Arg 1991; 8: 165-72.

19.- SCHANTZ P M, CRUZ REYES A, COLLI C, LORD R D. Sylvatic echinococcosis in Argentine. Tropenmed Parasitol 1975; 26: 334-44.

Agradecimientos: La Facultad de Ciencias Naturales de la Universidad Nacional de la Patagonia San Juan Bosco (U.N.P.S.J.B) subsidió en forma parcial el presente trabajo. La Sra. Yolanda Erkoreka y la Tecn. Quím. Roxana Silva (U.N.P.S.J.B) colaboraron en la recolección y procesamiento de muestras. 